\title{
Towards symbolic model checking for multi-agent systems via OBDD's
}

\author{
Franco Raimondi and Alessio Lomuscio \\ Department of Computer Science \\ King's College London \\ London, UK \\ \{franco,alessio\}@dcs.kcl.ac.uk
}

\begin{abstract}
We present an algorithm for model checking temporal-epistemic properties of multi-agent systems, expressed in the formalism of interpreted systems. We furst introduce a technique for the translation of interpreted systems into boolean formulae, and then present a modelchecking algorithm based on this translation. The algorithm is based on OBDD's, as they offer a compact and efficient representation for boolean formulae.
\end{abstract}

\section{Introduction}

Theoretical investigations in the area of multi-agent systems (MAS) have traditionally focused on specifications. Various logics have been explored to give formal foundations to MAS, particularly for mental attitudes [1] of agents, such as knowledge, belief, desire, etc. To consider the temporal evolution of these attitudes, temporal logics such as CTL and LTL [2] have been included in MAS formalisms, thereby producing combinations of temporal logic with, for example, epistemic, doxastic, and deontic logics.

Although it is important to investigate formal tools for specifying MAS, the problem of verification of MAS must also be taken into account to ensure that systems behave as they are supposed to. Model checking is a well-established verification technique for distributed systems specified by means of temporal logics $[3,2]$. The problem of model checking is to verify whether a logical formula $\varphi$ expressing a certain required property is true in a model $M$ representing the system, that is establishing whether or not $M \models \varphi$. This approach can also be applied to MAS, where in this case $M$ is a semantical model representing the evolutions of the MAS, and $\varphi$ is a formula expressing temporal-intentional properties of the agents. Recent work along these lines includes [4], in which Wooldridge et al. present the MABLE language for the specification of MAS. In this work, modalities are translated as nested data struciures (in the spirit of [5]). Bordini et al. [6] use a modified version of the AgentSpeak(L) language [7] to specify agents and to exploit existing model checkers. For verification purposes, both the works of Wooldridge et al. and of Bordini et al. translate the MAS specification into a SPIN specification [8] to perform the verification. The works 
of van der Meyden and Shilov [9], and van der Meyden and Su [10], are concerned with verification of interpreted systems. They consider the verification of a particular class of interpreted systems, namely the class of synchronous distributed systems with perfect recall. An algorithm for model checking is introduced in the first paper using automata, and [10] suggests the use of OBDD's for this approach.

The aim of this paper is to present an algorithm for model checking epistemic and temporal properties of interpreted systems [11]. This differs from previous work by treating all the modalities explicitly in the verification process. We focus on temporal-epistemic model checking because the verification of epistemic properties (and their temporal evolution) is crucial in many scenarios, including communication protocols and security protocols.

Interpreted systems are a formalism for representing epistemic properties of MAS and their evolution with time. The algorithm that we present does not involve the translation into existing model checkers, it is fully symbolic, and it is based on boolean functions. Boolean functions can be represented and manipulated efficiently by means of OBDD's, as it has been shown for CTL model checking [12].

The rest of the paper is organised as follows: in Section 2 we briefly review OBDD's-based model checking and the formalism of interpreted systems. In Section 3.1 we present the translation of interpreted systems into boolean formulae, while in Section 3.2 we introduce an algorithm based on this translation. We provide a proof of the correctness of the algorithm in Section 3.3. We conclude in Section 4.

\section{Preliminaries}

\subsection{CTL model checking and OBDD's}

Given a model $M$ and a formula $\varphi$ in some logic, the problem of model checking involves establishing whether or not $M \models \varphi$ holds. Tools have been built to perform this task automatically, where $M$ is a model of some temporal logic [3, $2,8]$. SMV [12] and SPIN [8] are two well-known model checkers; in these tools the model is given indirectly by means of a program $P$. It is not efficient to build explicitly the model $M$ represented by $P$, because $M$ has a size which is exponential in the number of variables of $P$ (this fact is known as the state explosion problem). Instead, various techniques have been developed to perform symbolic model checking, which is the problem of model checking where the model $M$ is not described or computed in extension. Techniques for symbolic model checking mostly use either automata [8], or OBDD's [13] for the representation of all the parameters needed by the algorithms. For the purpose of this paper, we will only consider symbolic model checking of the temporal logic CTL using OBDD's [14].

CTL is a logic used to reason about the evolution of a system represented as a branching path. Given a countable set of propositional variables $\mathcal{P}=\{p, q, \ldots\}$, CTL formulae are defined as follows:

$$
\varphi::=p|\neg \varphi| \varphi \vee \varphi|E X \varphi| E G \varphi \mid E(\varphi U \varphi)
$$


where the temporal operator $X$ means in the next state, $G$ means globally and $U$ means until. Each temporal operator is pre-fixed by the existential quantifier $E$. Thus, for example, $E G(\varphi)$ means that "there exists a path in which $\varphi$ is globally true". Traditionally, other operators are added to the syntax of CTL, namely $A X, E F, A F, A G, A U$ (notice the "universal" quantifier $A$ over paths, dual of $E)$. These operators can be derived from the operators introduced here [2]. The semantics of CTL is given via a model $M=(S, R, \mathcal{V}, I)$ where $S=\left\{s_{0}, s_{1}, \ldots\right\}$ is a set of states, $R \subseteq S \times S$ is a binary relation, $\mathcal{V}: \mathcal{P} \rightarrow 2^{S}$ is an evaluation function, and $I \subseteq S$ is a set of initial states. A path $\pi$ is a sequence of states $\pi=\left\{s_{0}, s_{1}, \ldots\right\}$ such that $s_{0} \in I$ and $\forall i,\left(s_{i}, s_{i+1}\right) \in R$. A state $s_{i}$ in a path $\pi$ is denoted with $\pi_{i}$. Satisfaction in a state is defined inductively as follows:

$$
\begin{array}{ll}
s \models p & \text { iff } s \in \mathcal{V}(p), \\
s \models E X \varphi & \text { iff there exists a path } \pi \text { such that } \pi_{i}=s \text { and } \pi_{i+1} \models \varphi, \\
s \models E G \varphi & \text { iff there exists a path } \pi \text { such that } \pi_{i}=s \text { and } \pi_{i+j} \models \varphi \\
& \quad \text { for all } j \geq 0 .
\end{array}
$$

$s \models E(\varphi U \psi)$ iff there exists a path $\pi$ such that $\pi_{i}=s$ and a $k \geq 0$ such that $\pi_{i+k} \models \psi$ and $\pi_{i+j} \models \varphi$ for all $0 \leq j<k$.

OBDD's (Ordered Binary Decision Diagrams) are an efficient representation for the manipulation of boolean functions. As an example, consider the boolean function $a \wedge(b \vee c)$. The truth table of this function would be 8 lines long. Equivalently, one can evaluate the truth value of this function by representing the function as a directed graph, as exemplified on the left-hand side of Figure 1. As it is clear from the picture, under certain assumptions, this graph can be simplified into the graph pictured on the right-hand side of Figure 1. This "reduced" representation is called the OBDD of the boolean function.

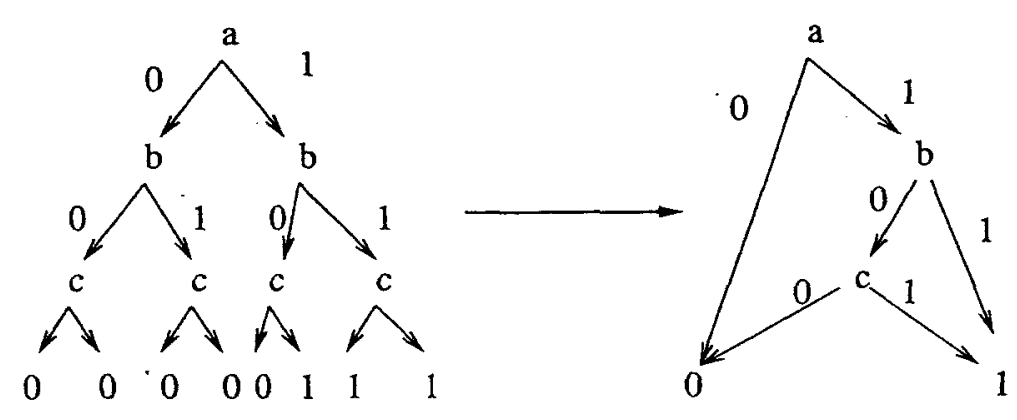

Fig. 1. OBDD representation for $a \wedge(b \vee c)$.

Besides offering a compact representation of boolean functions, OBDD's of different functions can be cumpuposed efficiently: in [13] algorithms are provided for the manipulation and composition of OBDD's.

The idea of CTL model checking using OBDD's is to represent states of the model and relations by means of boolean formulae. A CTL formula is identified with a set of states, i.e. the states of the model satisfying the formula. As set 
of states can be represented as a boolean formula, each CTL formula can be characterised by a boolean formula. Thus, the problem of model checking for CTL is reduced to the construction of boolean formulae. This is achieved by composing OBDD's, or by computing fix-points of operators on OBDD's; we refer to [2] for the details. By means of this approach large systems have been checked, including hardware and software components.

\subsection{Interpreted Systems}

An interpreted system is a semantic structure representing the temporal evolution of a system of agents. Each agent $i(i=\{1, \ldots, n\})$ is characterised by a set of local states $L_{i}$ and by a set of actions $A_{c} t_{i}$ that may be performed. Actions are performed in compliance with a protocol $P_{i}: L_{i} \rightarrow 2^{\text {Act }_{i}}$; notice that this definition allows for non-determinism. A tuple $g=\left(l_{1}, \ldots, l_{n}\right) \in L_{1} \times \ldots, L_{n}$, where $l_{i} \in L_{i}$ for each $i$, is called a global state and gives a snapshot of the system. Given a set $I$ of initial global states, the evolution of the system is described by $n$ evolution functions ${ }^{1}: t_{i}: L_{1} \times \ldots \times L_{n} \times A c t_{1} \times \ldots \times A c t_{n} \rightarrow L_{i}$ In this formalism the environment in which agents "live" is usually modeled by means of a special agent $E$; we refer to [11] for more details.

The set $I, t_{i}$ and the protocols $P_{i}$ generate a set of runs. Formally, a run $\pi$ is a sequence of global states $\pi=\left(g_{0}, g_{1}, \ldots\right)$ such that $g_{0} \in I$ and, for each pair $\left(g_{j}, g_{j+1}\right) \in \pi$, there exists a set of actions $a$ enabled by the protocols such that $t\left(g_{j}, a\right)=g_{j+1} . G \subseteq\left(L_{1} \times \ldots \times L_{n}\right)$ denotes the set of reachable global states.

Given a set of agents $A=\{1, \ldots, n\}$ with corresponding local states, protocols, and transition functions, a countable set of propositional variables $\mathcal{P}=$ $\{p, q, \ldots\}$, and a valuation function for the atoms $\mathcal{V}: \mathcal{P} \rightarrow 2^{G}$, an interpreted system is a tuple $I S=\left(G, I, \Pi, \sim_{1}, \ldots, \sim_{n}, \mathcal{V}\right)$. In the above $G$ is the finite set of reachable global states for the system, $I \subseteq G$ is the set of initial states, and $\Pi$ is the set of possible runs in the system. The binary relation $\sim_{i}, i \in A$, is defined by $g \sim_{i} g^{\prime}$ iff $l_{i}(g)=l_{i}\left(g^{\prime}\right)$, i.e. if the local state of agent $i$ is the same in $g$ and in $g^{\prime}$. Some issues arise with respect to the generation of the reachable states in the system given a set of protocols and transition relations; since they do not influence this paper we do not report them here.

Interpreted systems semantics can be used to interpret formulae of a temporal language enriched with epistemic operators [11]. Here we assume a temporal tree structure to interpret CTLK formulae [15]. The syntax of CTLK is defined in terms of a countable set of propositional variables $\mathcal{P}=\{p, q, \ldots\}$ and using the following modalities:

$$
\varphi::=p|\neg \varphi| \varphi \vee \varphi|E X \varphi| E G \varphi|E(\varphi U \varphi)| K_{i} \varphi
$$

The modalities $A X, E F, A F, A G, A U$ are derived in the standard way. Further, given a set of agents $\Gamma$, two group modalities can be introduced: $E_{\Gamma} \varphi$ and $C_{\Gamma} \varphi$ denote, respectively, that every agent in the group knows $\varphi$, and that $\varphi$ is common knowledge in the group (see [11] for details).

\footnotetext{
1 This definition is equivalent to the definition of a single evolution function $t$ as in [11].
} 
Given an interpreted system IS, a global state $g$, and a formula $\varphi$, the semantics of CTLK is defined as follows:

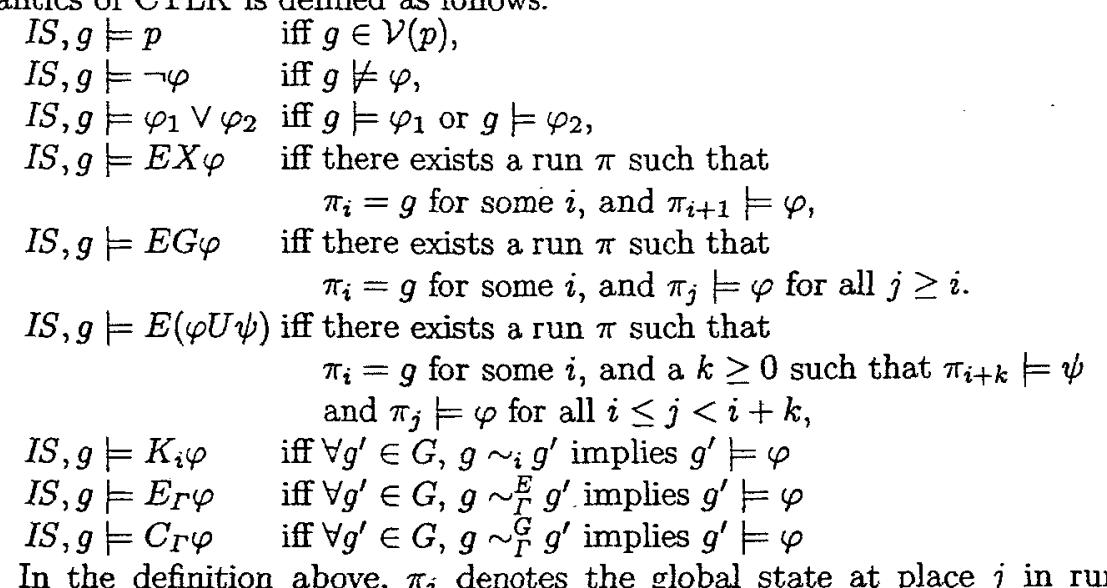

In the definition above, $\pi_{j}$ denotes the global state at place $j$ in run $\pi$. Other temporal modalities can be derived, namely $A X, E F, A F, A G, A \dot{U}$. We write $I S \models \varphi$ if, for every global state $g \in G, I S, g \models \varphi$. We refer to $[11,15]$ for more details.

\section{A model checking algorithm for CTLK}

The main idea of this paper is to use algorithms based on OBDD's to verify temporal and epistemic properties of multi-agent systems, in the spirit of traditional model checking for temporal logics. To this end, it is necessary to encode all the parameters needed by the algorithms by means of boolean functions, and then to represent boolean functions by means of OBDD's. As this last step can be performed automatically using software libraries that are widely available, in this paper we introduce only the translation of interpreted systems into boolean formulae (Section 3.1). In Section 3.2 we present an algorithm based on this translation for the verification of CTLK formulae.

\subsection{Translating an interpreted system into boolean formulae}

The local states of an agent can be encoded by means of boolean variables (a boolean variable is a variable that can assume just one of the two values 0 or 1). The number of boolean variables needed for each agent is $n v(i)=\left\lceil\log _{2}\left|L_{i}\right|\right\rceil$. Thus, a global state can be identified by means of $N=\sum_{i} n v(i)$ boolean variables: $g=\left(v_{1}, \ldots, v_{N}\right)$. The evaluation function $\mathcal{V}$ associates a set of global states to each propositional atom, and so it can be seen as a boolean function. The protocols, too, can be expressed as boolean functious (actions being represented with boolean variables $\left(a_{1}, \ldots, a_{M}\right)$ similarly to global states).

The definition of $t_{i}$ in Section 2.2 can be seen as specifying a list of conditions $c_{i, 1}, \ldots, c_{i, k}$ under which agent $i$ changes the value of its local state. Each $c_{i, j}$ relates conditions on global state and actions with the value of "next" local state 
for $i$.

$$
t_{i}=c_{i, 1} \vee \ldots \vee c_{i, k}
$$

We assume that the last condition $c_{i, k}$ of $t_{i}$ prescribes that, if none of the conditions $c_{i, j}(j<k)$ is true, then the local state for $i$ does not change. This assumption is key to keep compact the description of an interpreted system, as in this way only the conditions that are actually causing a change need to be listed.

The algorithm presented in Section 3.2 requires the definition of a boolean function $R_{t}\left(g, g^{\prime}\right)$ representing a temporal relation between $g$ and $g^{\prime} . R_{t}\left(g, g^{\prime}\right)$ can be obtained from the evolution function $t_{i}$ as follows. First, we introduce a global evolution function $t$ :

$$
t=\bigwedge_{i \in\{1, \ldots, n\}} t_{i}=\bigwedge_{i \in\{1, \ldots, n\}}\left(c_{i, 1} \vee \ldots \vee c_{i, k_{i}}\right)
$$

Notice that $t$ is a boolean function involving two global states and a joint action $a=\left(a_{1}, \ldots, a_{M}\right)$. To abstract from the joint action and obtain a boolean function relating two global states only, we can define $R_{t}$ as follows:

$R_{t}\left(g, g^{\prime}\right)$ iff $\exists a \in A c t: t\left(g, a, g^{\prime}\right)$ is true and each local action $a_{i} \in a$ is enabled by the protocol of agent $i$ in the local state $l_{i}(g)$.

The quantification over actions above can be translated into a propositional formula using a disjunction (see [12,3] for a similar approach to boolean quantification):

$$
R_{t}\left(g, g^{\prime}\right)=\bigvee_{a \in A c t}\left[\left(t\left(g, a, g^{\prime}\right) \wedge P(g, a)\right]\right.
$$

where $P(g, a)$ is a boolean formula imposing that the joint action $a$ must be consistent with the agents' protocols in global state $g . R_{t}$ gives the desired boolean relation between global states.

\subsection{The algorithm}

In this section we present the algorithm $S A T_{C T L K}$ to compute the set of global states in which a CTLK formula $\varphi$ holds, denoted with $[[\varphi]]$. The following are the parameters needed by the algorithm:

- the boolean variables $\left(v_{1}, \ldots, v_{N}\right)$ and $\left(a_{1}, \ldots, a_{M}\right)$ to encode global states and joint actions;

- the boolean functions $P_{i}\left(v_{1}, \ldots, v_{N}, a_{1}, \ldots, a_{M}\right)$ to encode the protocols of the agents;

- the function $\mathcal{V}(p)$ returning the set of global states in which the atomic proposition $p$ holds. We assume that the global states are returned encoded as a boolean function of $\left(v_{1}, \ldots, v_{N}\right)$;

- the set of initial states $I$, encoded as a boolean function;

- the set of reachable states $G$. This can be computed as the fix-point of the operator $\tau=\left(I(g) \vee \exists g^{\prime}\left(R_{t}\left(g^{\prime}, g\right) \wedge Q\left(g^{\prime}\right)\right)\right.$ where $I(g)$ is true if $g$ is an initial state and $Q$ denotes a set of global states. The fix-point of $\tau$ can be computed by iterating $\tau(\emptyset)$ by standard procedure (see [12]); 
The procedure $C_{C T L K}(\varphi, \Gamma)$ is based on the equivalence [11]

$$
C_{\Gamma} \varphi=E_{\Gamma}\left(\varphi \wedge C_{\Gamma} \varphi\right)
$$

which implies that $\left[\left[C_{\Gamma} \varphi\right]\right]$ is the fix-point of the (monotonic) operator $\tau(Q)=$ $\left[\left[E_{\Gamma}(\varphi \wedge(Q))\right]\right]$. Hence, $\left[\left[C_{\Gamma} \varphi\right]\right]$ can be obtained by iterating $\tau(G)$.

Notice that all the parameters can be encoded as OBDD's. Moreover, all the operations inside the algorithms can be performed on OBDD's as presented in [13].

To check that a formula holds in a model, it is enough to check whether or not the result of $S A T_{C T L K}$ is equivalent to the set of reachable states.

\subsection{Correctness of the algorithm}

The algorithm presented in Section 3.2 is sound and complete.

Theorem 1. For every CTLK formula $\varphi$, IS $\models \varphi$ iff $S A T_{C T L K}(\varphi) \equiv G$. (i.e. iff the set of states computed by the algorithm is the set of reachable states $G$ ).

Proof. (=>): by induction on the structure of $\varphi$. We consider here the epistemic operators (a proof for the temporal operators can be found in [2]). Let $\varphi=K_{i}(\psi)$ and let $I S, g \models K_{i}(\psi)$. This means that $I S, g^{\prime} \models \psi$ for all $g^{\prime} \in G$ s.t. $g \sim_{i} g^{\prime}$. By the induction step, $g^{\prime} \in[[\psi]]$; also we have $R_{i}\left(g, g^{\prime}\right)$ by definition of $R_{i}$. This implies that $g \in\left[\left[K_{i}(\psi)\right]\right]$, i.e. $g \in[[\varphi]]$. The proof for $E_{\Gamma}$ is similar. The proof of correctness for common knowledge follows from the correctness of the fix-point characterisation of $C_{\Gamma}[11]$.

$(<=)$ : straightforward, as the induction steps above are symmetrical.

\section{Conclusion}

Temporal logic model checking using OBDD's [12] is one of the most successful techniques for the verification of distributed systems. In the last decade, this methodology has been used for the verification of both software and hardware components.

In this paper we have presented an algorithm for the verification of temporalepistemic properties based on the manipulation of boolean functions. The methodology presented here encodes directly a MAS (specified in the formalism of interpreted systems) by means of boolean formulae; then, the algorithm allows for the (fully symbolic) verification of temporal-epistemic properties. Moreover, the algorithm allows for the verification of two group modalities $\left(E_{\Gamma}\right.$ and $\left.C_{\Gamma}\right)$ and is not restricted to a particular class of interpreted systems, nor to a particular class of formulae. We are currently implementing the algorithm and in the future we aim at testing epistemic and temporal properties of various scenarios from the MAS literature. This will help in evaluating the efficiency of the algorithm. 


\section{References}

1. McCarthy, J.: Ascribing mental qualities to machines. In Ringle, M., ed.: Philosophical Perspectives in Artificial Intelligence. Humanities Press, Atlantic Highlands, New Jersey (1979) 161-195

2. Huth, M.R.A., Ryan, M.D.: Logic in Computer Science: Modelling and Reasoning about Systems. Cambridge University Press, Cambridge, England (2000)

3. Clarke, E.M., Grumberg, O., Peled, D.A.: Model Checking. The MT Press, Cambridge, Massachusetts (1999)

4. Wooldridge, M., Fisher, M., Huget, M.P., Parsons, S.: Model checking multiagent systems with MABLE. In Gini, M., Ishida, T., Castelfranchi, C., Johnson, W.L., eds.: Proceedings of the First International Joint Conference on Autonomous Agents and Multiagent Systems (AAMAS'02), ACM Press (2002) 952-959

5. Benerecetti, M., Giunchiglia, F., Serafini, L.: Model checking multiagent systems. Journal of Logic and Computation 8 (1998) 401-423

6. Bordini, R.H., Fisher, M., Pardavila, C., Wooldridge, M.: Model checking AgentSpeak. In: Proceedings of the Second International Joint Conference on Autonomous Agents and Multiagent Systems (AAMAS'03). (2003)

7. Rao, A.S.: AgentSpeak(L): BDI agents speak out in a logical computable language. Lecture Notes in Computer Science 1038 (1996) 42-58

8. Holzmann, G.J.: The model checker spin. IEEE transaction on software engineering 23 (1997)

9. van der Meyden, R., Shilov, N.V.: Model checking knowledge and time in systems with perfect recall. FSTTCS: Foundations of Software Technology and Theoretical Computer Science 19 (1999)

10. van der Meyden, R., Su, K.: Symbolic model checking the knowledge of the dining cryptographers. Submitted (2002)

11. Fagin, R., Halpern, J.Y., Moses, Y., Vardi, M.Y.: Reasoning about Knowledge. The MIT Press, Cambridge, Massachusetts (1995)

12. McMillan, K.: Symbolic model checking: An approach to the state explosion problem. Kluwer Academic Publishers (1993)

13. Bryant, R.E.: Graph-based algorithms for boolean function manipulation. IEEE Transaction on Computers (1986) 677-691

14. Burch, J.R., Clarke, E.M., McMillan, K.L., Dill, D.L., Hwang, L.J.: Symbolic model checking: $10^{20}$ states and beyond. Information and Computation 98 (1992) $142-170$

15. Penczek, W., Lomuscio, A.: Verifying epistemic properties of multi-agent systems via model checking. Fundamenta Informaticae 55 (2003) 167-185 